\title{
openheart Catheter ablation and mortality, stroke and heart failure readmission with atrial fibrillation
}

Jannik Langtved Pallisgaard (D , , ${ }^{1}$ Morten Lock Hansen, ${ }^{1}$ Anne-Marie Schjerning, ${ }^{1}$ Arne Johannessen, ${ }^{1}$ Thomas Alexander Gerds, ${ }^{2}$ Finn Gustafsson, ${ }^{3}$ Gunnar Hilmar Gislason, ${ }^{1}$ Chriatian Torp-Pedersen, ${ }^{4}$ Peter Karl Jacobsen, ${ }^{3}$ Søren Lund Kristensen, ${ }^{1}$ Lars Koeber, ${ }^{3}$ Anders Munch, ${ }^{2}$ Morten Schou ${ }^{5}$

- Additional material is published online only. To view, please visit the journal online (http://dx.doi.org/10.1136/ openhrt-2020-001369).

To cite: Pallisgaard JL, Lock Hansen M, Schjerning A-M, et al. Catheter ablation and mortality, stroke and heart failure readmission with atrial fibrillation. Open Heart 2020;7:e001369. doi:10.1136/ openhrt-2020-001369

Received 24 June 2020 Revised 15 0ctober 2020 Accepted 19 0ctober 2020
Check for updates

(c) Author(s) (or their employer(s)) 2020. Re-use permitted under CC BY-NC. No commercial re-use. See rights and permissions. Published by BMJ.

For numbered affiliations see end of article.

\section{Correspondence to}

Dr Jannik Langtved Pallisgaard; jannikjannik@gmail.com

\section{ABSTRACT}

Background Recent randomised clinical trials have suggested prognostic benefits of catheter ablation in highly selected patients with atrial fibrillation (AF) and heart failure (HF).

Objectives This study sought to identify the treatment effect associated with catheter ablation in a broad population of patients with $\mathrm{AF}$ and $\mathrm{HF}$.

Methods Through nationwide administrative registers in Denmark, we estimated the 2-year average treatment effect (ATE) of catheter ablation for AF on a composite endpoint of HF readmission, stroke and all-cause mortality at 1-year and 5-year landmark analyses. The primary cohort was patients with AF before HF, and the second cohort of patients with $\mathrm{HF}$ before AF.

Results A total of 13756 patients were included with 9904 patients in the primary cohort, and 3852 in the secondary. An ATE $(95 \% \mathrm{Cl})$ reduction of the composite endpoint of $7.0 \%$ (4.5\% to $9.5 \%)$ was observed in the primary cohort and $11.8 \%(6.0 \%$ to $17.6 \%)$ in the secondary in the 1-year landmark analysis with a reduction in all-cause mortality of $5.8 \%(3.7 \%-7.8 \%)$ and $6.3 \%(0.9 \%-11.7 \%)$, respectively. At the 5 -year landmark, catheter ablation was associated with reductions in the composite endpoint and all-cause mortality in the primary $(4.7 \%(2.3 \%$ to $7.2 \%)$, and $3.6 \%(1.0 \%$ to $6.3 \%)$, respectively), but not in the secondary cohort.

Conclusions Ablation was associated with decreased risk of $\mathrm{HF}$ readmission, stroke and all-cause mortality in patients with AF and HF. The effect is most substantial in patients with $\mathrm{AF}$ before HF and with catheter ablation after 1 year from the diagnosis of both conditions.

\section{INTRODUCTION}

Pharmacological treatment of atrial fibrillation (AF) in patients with concomitant heart failure (HF) poses a challenge in daily clinical practice. ${ }^{1}$ Medical therapy remains the first choice in the management of $\mathrm{AF}$, but modest efficacy, adverse effects and contraindications are significant limitations to this strategy. ${ }^{2}$ Catheter ablation to restore sinus rhythm is, therefore, an intriguing additional treatment option, especially in patients with

\section{Key questions}

What is already known about this subject?

- Recent studies have shown a possible decreased risk of death with radiofrequency ablation in patients with atrial fibrillation and heart failure. Atrial fibrillation can lead to tachycardia-induced heart failure, but also heart failure increases the risk of developing atrial fibrillation.

What does this study add?

- Our study shows a decreased risk of readmission with heart failure, stroke and all-cause mortality with radiofrequency ablation in both patients with atrial fibrillation before heart failure and in patients with heart failure before atrial fibrillation. This reduction was most prominent in patients with tachycardiainduced heart failure.

How might this impact on clinical practice?

- Radiofrequency ablation should not only be seen as symptom-relieving but also as a potential mortalityreducing procedure in patients with atrial fibrillation and heart failure.

a tachyarrhythmia-induced cardiomyopathy. Small studies have suggested that catheter ablation may improve left ventricular function and functional capacity in patients with $\mathrm{AF}$ and $\mathrm{HF}^{4-7}$ and recently randomised controlled trials reported that catheter ablation might reduce the risk of admission for $\mathrm{HF}$ and mortality. ${ }^{89}$

Considering the neutral result of the AF-CHF trial (Rhythm Control vs Rate Control for AF and HF), the mortality benefit observed in the CASTLE-AF trial (Catheter Ablation vs Standard Conventional Treatment in Patients with Left Ventricular Dysfunction and Atrial Fibrillation) was somewhat surprising. ${ }^{10}$ It has been hypothesised that small study size in CASTLE-AF (ie, type I error), lack of balancing of baseline variables, an uneven number of patients lost to follow-up, as well 
as bias due to an inherent open study design may explain the results. ${ }^{11}$ Finally, it is unclear how the results from too slow recruiting trials evaluating treatment effect in typical conditions $(20 \%-40 \%$ of all patients with HF have $\mathrm{AF})$ should be translated into clinical practice. For these reasons, patient selection for catheter ablation remains unclear in patients with $\mathrm{AF}$ and concomitant HF.

The present study sought to evaluate the real-life association between $\mathrm{AF}$ catheter ablation and a composite endpoint of stroke, admission for HF or all-cause mortality in a nationwide cohort of patients with $\mathrm{AF}$ and $\mathrm{HF} \leq 75$ years and all treated with oral anticoagulation. Analyses were done separately for patients with preexisting AF before $\mathrm{HF}$ diagnosis and those with $\mathrm{AF}$ after HF diagnosis. The rationale for the two separate cohorts was the preconceived notion that patients with $\mathrm{AF}$ before HF would have tachyarrhythmia-induced HF and, therefore, more significant benefit from catheter ablation than patients with $\mathrm{HF}$ before $\mathrm{AF}$, where structural myocardial heart disease and increased left ventricular filling pressures more likely would be the pathogenesis for AF.

\section{METHODS}

\section{Data sources}

In this register-based cohort study, information on demographics, comorbidities, procedures, concomitant medication, and outcome variables were identified using three different nationwide Danish registers. These registers were cross-linked using the unique personal identification number given to all Danish citizens at the date of birth or date of migration to Denmark. The Civil Registration System holds data on age, sex and vital status of patients, where all deaths are registered within 14 days of occurrence. The Danish National Patient Register contains information on every hospital admission in Denmark since 1978, in which each hospitalisation is registered at discharge with one primary diagnosis and, if applicable, one or more secondary diagnoses according to the International Classification of Diseases; the 10th revision (ICD-10), since 1994. The Danish National Patient Register also holds information on operations and procedures, including catheter ablation. These procedures have been registered since 1996 and coded according to the Nordic Classification of Surgical Procedures (NCSP) by The Nordic Medico-Statistical Committee. Data on the date, quantity, strength, formulation and affiliation of the prescribing physician, all prescriptions dispensed from Danish pharmacies have been accurately registered in The Danish Registry of Medicinal Product Statistics since 1995 and coded according to the Anatomical Therapeutic Chemical classification system.

Prevalent AF was identified using ICD-10 code 'I48' in both inpatients and outpatients. Patients with $\mathrm{HF}$ were identified based on our hospital outpatient clinic discharge diagnostic codes. Prevalent HF was identified using the ICD-10 code 'I50', 'I11.0', 'I13.0' or 'I13.2', also in both inpatients and outpatients. ${ }^{13}$ Catheter ablation procedures for AF were identified using the NCSP procedure code 'BFFB04' for AF catheter ablation (online supplemental table 1).

\section{Study cohort}

Patients were included between 2005 and 2017, age ranged between 18 and 75 years, no history of prior catheter ablation, or ischaemic stroke and were all treated with oral anticoagulants. The age and anticoagulation criteria were used to include a study population more likely to be referred to an AF catheter ablation. The analyses were done separately in two cohorts: the primary cohort contained patients who were diagnosed with $\mathrm{AF}$ before or the same day as HF, and the secondary cohort included patients with HF before AF.

Patients in the primary cohort were followed from the date of HF diagnosis, and for the secondary cohort date of AF diagnosis. This date is referred to as index date in the two cohorts, respectively.

\section{Study endpoint}

The primary endpoint was a composite of HF readmission, stroke and all-cause mortality, and secondary endpoints were the endpoints separately.

\section{Patient and public involvement statement}

The patients and public were not involved in the creation of the study design or statistical analysis. Patients were not consulted to develop patient-relevant outcomes or interpret the results. Patients were not invited to contribute to the writing or editing of this document for readability or accuracy.

\section{STATISTICS}

All analyses were performed separately, starting from two landmark time points set at 1 year and 5 years after study inclusion, respectively. Patients were included if they were alive and without events at landmark. The two landmark analyses were performed at 1 and 5 years in order to investigate whether potential benefits of catheter ablation would be present in both patients with long and short duration of $\mathrm{AF}$ and $\mathrm{HF}$ prior to the procedure. Patients were then subdivided according to whether they had received catheter ablation in the time between the index date and the landmark date. Patients who had an event included in the primary composite endpoint between the date of HF diagnosis and landmark date were excluded from the analyses, in order to avoid immortal time bias.

Patients were followed from the landmark time point until the composite endpoint, date of emigration or 1 January 2017, whatever came first (figure 1). The absolute personalised risks of the composite endpoint within the first 2 years after the landmark time point were estimated using Cox regression adjusted for patient characteristics evaluated at the landmark time point: catheter ablation status (yes/no) and the predictor variables of chronic obstructive pulmonary disease (COPD), chronic kidney disease $(\mathrm{CKD})$, ischaemic heart disease (IHD), diabetes 


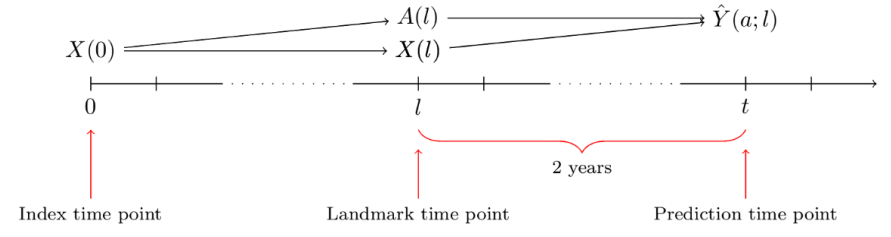

Figure 1 Flowchart of the landmark analyses. Patients were then followed from the landmark time point until the composite endpoint, date of emigration or 1 January 2017 whatever came first.

mellitus (DM), hypertension, outpatient HF diagnosis (yes/no), age and sex.

Average treatment effects (ATEs) were defined as differences between the standardised absolute risks of the combined endpoint occurring within 2 years after the landmark time. ${ }^{14}$ Analyses were repeated with allcause mortality as an outcome instead of the composite endpoint. For the two outcomes readmission with HF and stroke, death without readmission for HF (without stroke) is a competing risk. We used the cause-specific Cox regression approach of Benichou and Gail ${ }^{15}$ to estimate the absolute personalised risks of HF readmission, respectively, admission with stroke, within 2 years after the landmark date (online supplemental figure 1).

Categorical data were presented as counts with percentages, and the statistical difference was tested using $\chi^{2}$ tests. Continuous variables were presented as medians

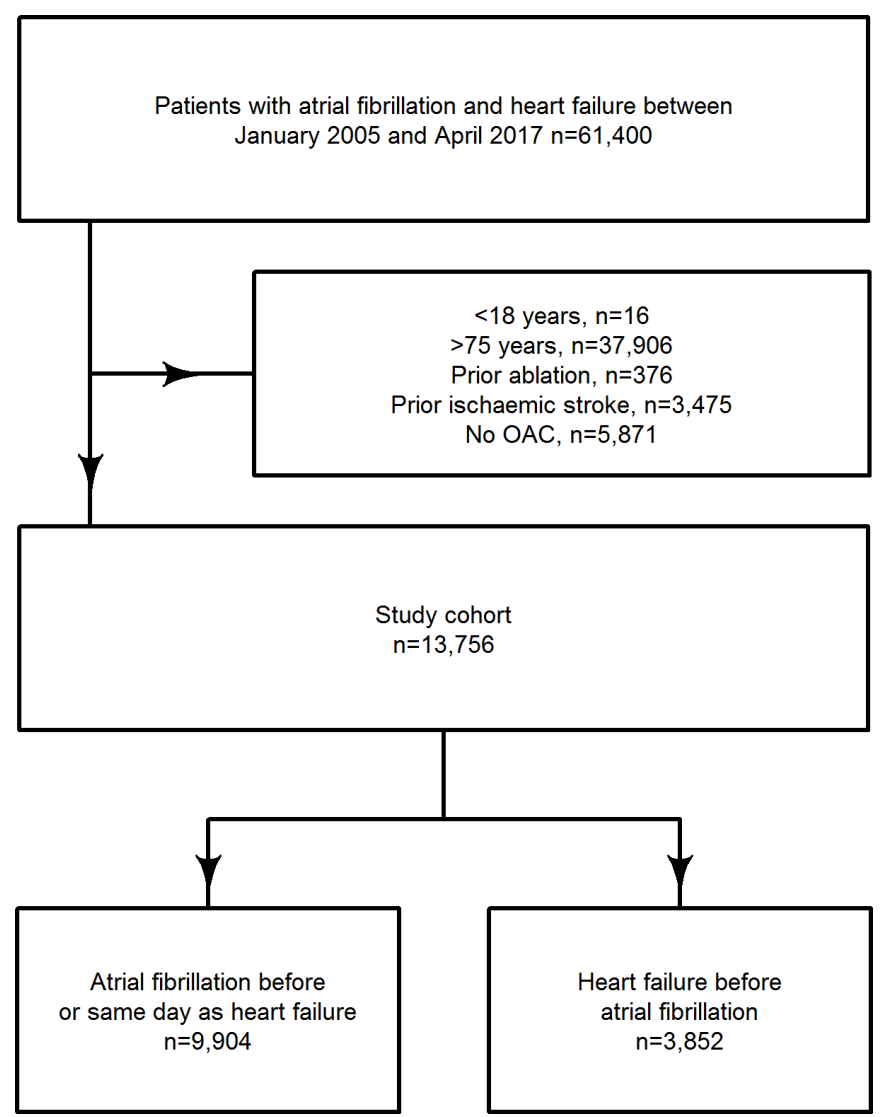

Figure 2 Flowchart of the study cohort selection. OAC, oral anticoagulation. with IQRs, and the statistical difference was tested using Wilcoxon rank-sum tests. Logistic regression (propensity score model) was used to associate patient characteristics evaluated at the index date with the odds of receiving catheter ablation within the landmark date. Data management and statistical analyses were conducted using $\mathrm{R}$ statistics. ${ }^{16}$ A p value $<0.05$ and a $95 \%$ CI not containing 1 was considered significant.

\section{RESULTS}

The total number of patients included in the study were 13756 with $9904(72 \%)$ in the primary cohort and 3852 $(28 \%)$ in the secondary cohort. Of the 9904 patients in the primary cohort (AF before HF), 3677 (37.1\%) were diagnosed with $\mathrm{AF}$ and $\mathrm{HF}$ on the same day

(figure 2). At baseline, the median (IQR) age was 67 (61-71) years, and $74.2 \%$ were men with no difference found between the two cohorts, but significantly more comorbidities were observed in the second cohort (table 1).

After 1 year, $259(3.1 \%)$ and $50(1.6 \%)$ patients had received a catheter ablation in the primary and secondary cohort, respectively. The total number of catheter ablations performed in the study period was $749(5.4 \%)$, with $627(6.3 \%)$ in the primary cohort and $122(3.2 \%)$ in the secondary. Higher age, COPD, IHD, DM all significantly decreased the likelihood of a catheter ablation (table 2 and figure 3 ).

The absolute risk difference as defined by the ATE (95\% CI) in patients who received catheter ablation compared with those who did not after 1 year was $7.0 \%$ $(4.5 \%-9.5 \%)$ in the primary cohort and $11.8 \%(6.0 \%-$ $17.6 \%$ ) in the secondary cohort regarding the composite endpoint. The composite endpoint was mainly driven by all-cause mortality and readmission with $\mathrm{HF}$ in both cohorts. The ATE $(95 \% \mathrm{CI})$ of all-cause mortality after 1 year was $5.8 \%(3.7 \%-7.8 \%)$ and $6.3 \%(0.9 \%-11.7 \%)$ for the primary cohort and secondary cohort, respectively.

After 5 years, $286(7.1 \%)$ and $42(3.3 \%)$ of the eligible patients had received catheter ablation in the primary and secondary cohort, respectively, with the same selection towards younger age and fewer comorbidities, as seen in the 1-year landmark analyses (table 3 ).

The ATE (95\% CI) of catheter ablation on the composite endpoint was still significant, with $4.7 \%(2.3 \%-7.2 \%)$ in the primary cohort with an ATE (95\% CI) of catheter ablation on all-cause mortality of $3.6 \%(1.0 \%-6.3 \%)$. Still, in the second cohort, a significant ATE was no longer observed $2.8 \%$ ( $-7.8 \%$ to $13.2 \%$, figure 4$)$.

\section{DISCUSSION \\ Main findings}

In this nationwide study, we found a significant association between AF catheter ablation and a reduced risk of the composite endpoint of HF readmission, stroke and all-cause mortality. Five years after the index date, the effect of was attenuated, and AF catheter ablation was no 


\begin{tabular}{|c|c|c|c|c|}
\hline Variable & AF before HF $(n=9904)$ & HF before AF ( $n=3852)$ & Total cohort $(n=13756)$ & $P$ value \\
\hline Age, median (IQR) & $66.9(61-71)$ & $66.9(61-71)$ & $66.9(61-71)$ & 0.7132 \\
\hline Men, n (\%) & 7320 (73.9) & $2885(74.9)$ & 10205 (74.2) & 0.2438 \\
\hline COPD, n (\%) & $1474(14.9)$ & $831(21.6)$ & 2305 (16.8) & $<0.001$ \\
\hline CKD, n (\%) & $554(5.6)$ & $391(10.2)$ & $945(6.9)$ & $<0.001$ \\
\hline IHD, n (\%) & 3031 (30.6) & $2034(52.8)$ & $5065(36.8)$ & $<0.001$ \\
\hline DM, n (\%) & $1885(19.0)$ & $1057(27.4)$ & $2942(21.4)$ & $<0.001$ \\
\hline HT, n (\%) & 9553 (96.5) & $3775(98.0)$ & $13328(96.9)$ & $<0.001$ \\
\hline Outpatients & $2654(26.8)$ & $1285(33.4)$ & $3939(28.6)$ & $<0.001$ \\
\hline Thiazides, $n(\%)$ & $1640(16.6)$ & $561(14.6)$ & $2201(16.0)$ & 0.005 \\
\hline Spironolactone, $\mathrm{n}(\%)$ & $2627(26.5)$ & $1456(37.8)$ & $4083(29.7)$ & $<0.001$ \\
\hline Loop, n (\%) & $6918(69.9)$ & $2739(71.1)$ & 9557 (70.2) & 0.154 \\
\hline Beta-blocker, n (\%) & $8367(84.5)$ & $3193(82.9)$ & $11560(84.0)$ & 0.024 \\
\hline Calcium channel blocker, $\mathrm{n}(\%)$ & $2604(26.3)$ & $863(22.4)$ & 3467 (25.2) & $<0.001$ \\
\hline RAS inhibitor, n (\%) & $7724(78.0)$ & $3165(82.2)$ & 10889 (79.2) & $<0.001$ \\
\hline Verapamil, n (\%) & $784(7.9)$ & $145(3.8)$ & $929(6.8 \%)$ & $<0.001$ \\
\hline Amiodarone, $\mathrm{n}(\%)$ & 1625 (16.4) & $643(16.7)$ & 2268 (16.5) & 0.682 \\
\hline Digoxin, n (\%) & $5091(51.4)$ & $1569(40.7)$ & $6660(48.4)$ & $<0.001$ \\
\hline Dabigatran, n (\%) & $1124(11.3)$ & $367(9.5)$ & $1491(10.8)$ & 0.002 \\
\hline Rivaroxaban, n (\%) & $651(6.6)$ & $255(6.6)$ & $906(6.6)$ & 0.951 \\
\hline Apixaban, n (\%) & $750(7.6)$ & $347(9.0)$ & $1097(8.0)$ & 0.006 \\
\hline Warfarin, n (\%) & 7550 (76.2) & 2935 (76.2) & 10485 (76.2) & 0.981 \\
\hline Marcumar, n (\%) & $124(1.3)$ & $48(1.2)$ & $172(1.3)$ & 1.000 \\
\hline ICD, n (\%) & $339(3.4)$ & $526(13.7)$ & $865(6.3)$ & $<0.001$ \\
\hline $\mathrm{PCl}, \mathrm{n}(\%)$ & $940(9.5)$ & $783(20.3)$ & $1723(12.5)$ & $<0.001$ \\
\hline CABG, $n(\%)$ & $631(6.4)$ & $584(15.2)$ & $1215(8.8)$ & $<0.001$ \\
\hline CHA2DS2-VASc (IQR) & $3(2-4)$ & $3(2-4)$ & $3(2-4)$ & $<0.001$ \\
\hline
\end{tabular}

The distribution of the predictor variables at the index date.

CABG, coronary artery bypass graft; CKD, chronic kidney disease; COPD, chronic obstructive pulmonary disease; DM, diabetes mellitus; $\mathrm{HT}$, hypertension; ICD, implantable cardioverter-defibrillator; IHD, ischaemic heart disease; PCl, percutaneous coronary intervention.

Table 2 Patients eligible for 1-year landmark analysis

\begin{tabular}{|c|c|c|c|c|c|c|}
\hline \multirow[b]{2}{*}{ Variable } & \multicolumn{3}{|c|}{ AF before HF } & \multicolumn{3}{|c|}{ HF before AF } \\
\hline & $\begin{array}{l}\text { No-catheter } \\
\text { ablation }(n=8154)\end{array}$ & $\begin{array}{l}\text { Catheter ablation } \\
(n=259)\end{array}$ & $P$ value & $\begin{array}{l}\text { No-catheter } \\
\text { ablation }(n=3052)\end{array}$ & $\begin{array}{l}\text { Catheter ablation } \\
(n=50)\end{array}$ & $P$ value \\
\hline Age median (IQR) & $67.7(62.0-71.9)$ & $61.1(52.6-66.9)$ & $<0.001$ & $67.6(61.4-71.9)$ & 61.9 (51.9-66.0) & $<0.001$ \\
\hline Sex male & 6051 (74.2) & 210 (81.1) & 0.015 & $2279(74.7)$ & $42(84.0)$ & 0.179 \\
\hline COPD & $1303(16.0)$ & $21(8.1)$ & $<0.001$ & 656 (21.5) & $5(10.0)$ & 0.073 \\
\hline CKD & $520(6.4)$ & $10(3.9)$ & 0.131 & $315(10.3)$ & $<3(4.0)$ & 0.219 \\
\hline $\mathrm{IHD}$ & 2858 (35.1) & $70(27.0)$ & 0.009 & $1665(54.6)$ & $24(48.0)$ & 0.435 \\
\hline DM & 1747 (21.4) & $33(12.7)$ & 0.001 & $842(27.6)$ & $11(22.0)$ & 0.473 \\
\hline HT & 8048 (98.7) & 255 (98.5) & 0.950 & 3015 (98.8) & $49(98.0)$ & 1.000 \\
\hline Outpatient & $2290(28.1)$ & $60(23.2)$ & 0.096 & $1052(34.5)$ & $26(52.0)$ & 0.015 \\
\hline
\end{tabular}

The distribution of the predictor variables at the 1-year landmark.

$\mathrm{AF}$, atrial fibrillation; CKD, chronic kidney disease; COPD, chronic obstructive pulmonary disease; DM, diabetes mellitus; HF, heart failure; $\mathrm{HT}$, hypertension; IHD, ischaemic heart disease. 


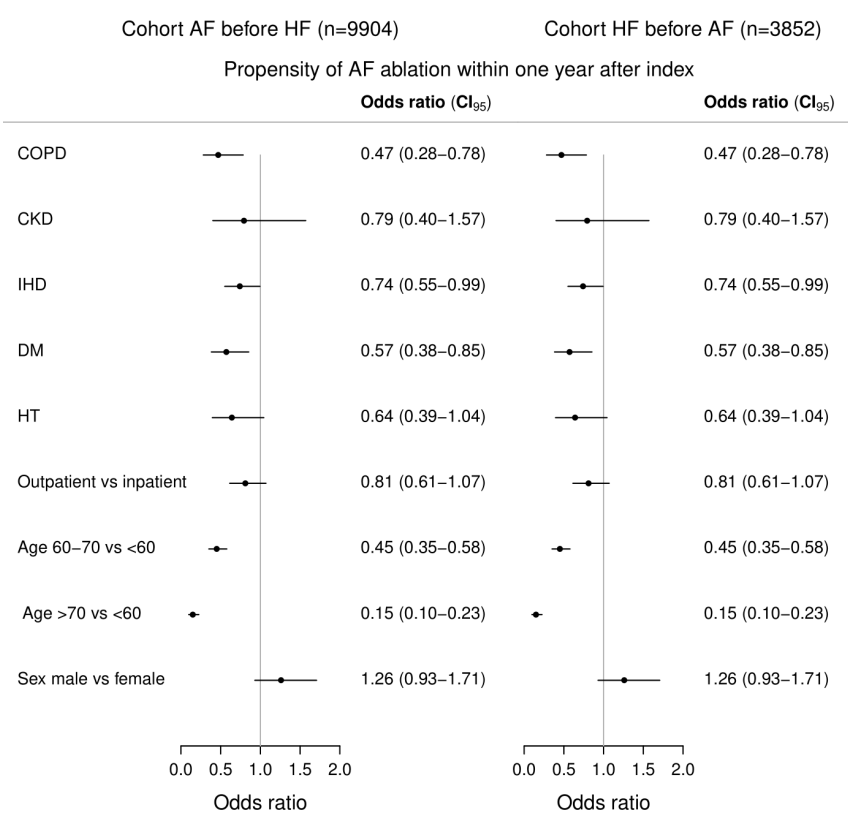

Figure 3 Propensity of predictor variables towards catheter ablation. AF, atrial fibrillation; COPD, chronic obstructive pulmonary disease; CKD, chronic kidney disease; DM, diabetes mellitus; HF, heart failure; HT, hypertension; IHD, ischaemic heart disease.

longer associated with a reduced risk of $\mathrm{HF}$ readmission or stroke.

\section{Real-life effect versus the clinical trial effect of catheter ablation in HF}

In the CASTLE-AF trial, a $16.1 \%(28.5 \%$ vs $44.6 \%$, p value $=0.007$ ) absolute risk reduction of death from any cause or hospitalisation for worsening HF was found with catheter ablation versus medical therapy after a median follow-up of 3 years. ${ }^{9}$ In our real-life study, we observed an estimated ATE of $7.0 \%$ after 2 years follow-up and $4.7 \%$ after 5 years in the primary cohort and $11.8 \%$ and $2.8 \%$ (non-significant) in the secondary cohort. Several factors may explain this discrepancy. Stroke was not
AF before HF $(n=9904) \quad$ HF before AF $(n=3852)$

n ATE $\left(\mathrm{Cl}_{95}\right)$ n ATE $\left(\mathrm{Cl}_{95}\right)$ One year after index

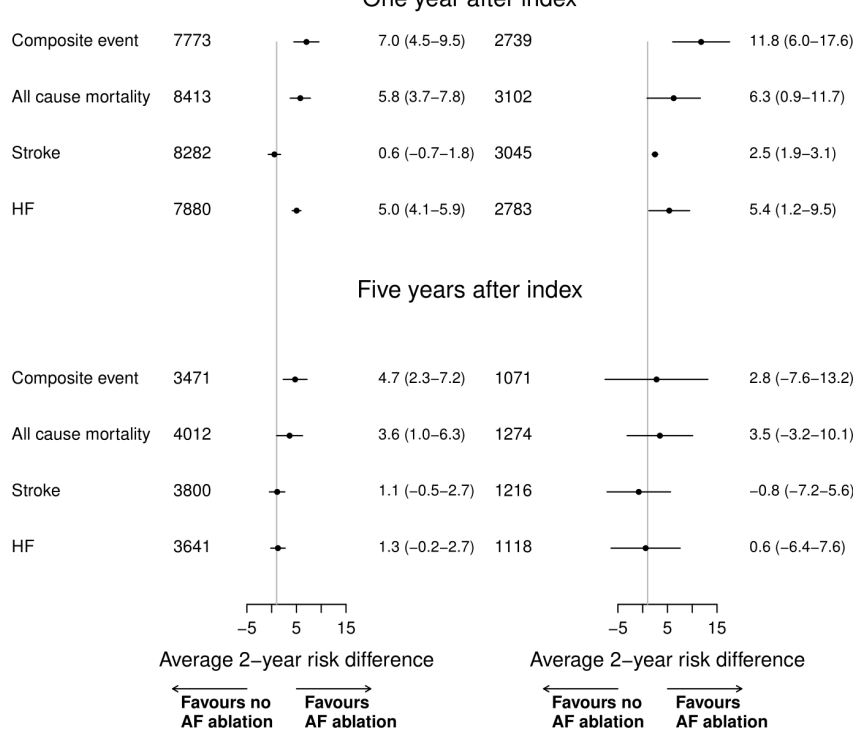

Figure 4 Central illustration. ATE of catheter ablation on endpoints. In the 1-year analyses, 259 (3.1\%) and 50 $(1.6 \%)$ had an ablation in the primary and secondary cohort, respectively, this was $286(7.1 \%)$ and $42(3.3 \%)$ in the 5-year analyses. AF, atrial fibrillation; ATE, average treatment effect; $\mathrm{HF}$, heart failure.

included as an endpoint in the in CASTLE-AF study. The median age in CASTLE-AF was 64 years compared with 70 in our study, $86 \%$ were men compared with $74 \%$ in our cohort, and the number of patients with IHD was $46 \%$ in CASTLE-AF compared with $37 \%$ in our study. We included patients with HF with both preserved and reduced left ventricular ejection fraction and patients without an implantable cardioverter-defibrillator (ICD). In the CASTLE-AF study, only patients with HFrEF (HF with reduced ejection fraction) and ICD were included.

In the CABANA trial, the primary endpoint was a composite of death, disabling stroke, severe bleeding

Table 3 Patients eligible for 5-year landmark analysis

\begin{tabular}{|c|c|c|c|c|c|c|}
\hline \multirow[b]{2}{*}{ Variable } & \multicolumn{3}{|l|}{ AF before HF } & \multicolumn{3}{|l|}{ HF before AF } \\
\hline & $\begin{array}{l}\text { No-catheter } \\
\text { ablation }(n=3726)\end{array}$ & $\begin{array}{l}\text { Catheter ablation } \\
(n=286)\end{array}$ & $P$ value & $\begin{array}{l}\text { No-catheter } \\
\text { ablation }(n=1232)\end{array}$ & $\begin{array}{l}\text { Catheter ablation } \\
(n=42)\end{array}$ & $P$ value \\
\hline Age median (IQR) & $70.8(65.0-75.4)$ & $64.9(58.5-69.6)$ & $<0.001$ & $70.3(64.4-75.2)$ & $62.4(56.2-68.3)$ & $<0.001$ \\
\hline Sex male & 2732 (73.3) & $229(80.1)$ & 0.015 & $916(74.4)$ & $29(69.0)$ & 0.553 \\
\hline COPD & $612(16.4)$ & $30(10.5)$ & 0.011 & 261 (21.2) & $3(7.1)$ & 0.044 \\
\hline CKD & 295 (7.9) & $8(2.8)$ & 0.002 & $142(11.5)$ & $3(7.1)$ & 0.527 \\
\hline IHD & 1509 (40.5) & 107 (37.4) & 0.335 & $687(55.8)$ & $16(38.1)$ & 0.035 \\
\hline DM & $971(26.1)$ & 41 (14.3) & $<0.001$ & $362(29.4)$ & $15(35.7)$ & 0.476 \\
\hline HT & 3692 (99.1) & $286(100.0)$ & 0.198 & 1222 (99.2) & $42(100.0)$ & 1.000 \\
\hline Outpatient & 1064 (28.6) & 67 (23.4) & 0.073 & $433(35.1)$ & $16(38.1)$ & 0.819 \\
\hline
\end{tabular}

The distribution of the predictor variables at the 5-year landmark.

$\mathrm{AF}$, atrial fibrillation; CKD, chronic kidney disease; COPD, chronic obstructive pulmonary disease; DM, diabetes mellitus; HF, heart failure; HT, hypertension; IHD, ischaemic heart disease. 
or cardiac arrest. In the intention-to-treat analysis of the subgroup with congestive HF ( $15 \%$ of the total cohort), a non-significant $5.7 \%$ reduction of the primary endpoint occurred in the catheter ablation group $(12.1 \%$ vs $17.8 \%, \mathrm{p}$ value $=\mathrm{NS}$ ) versus the medical therapy group over a median follow-up of 4 years. ${ }^{17}$ The findings in the CABANA trial are closer to the conclusions of our observational study, although we found a significant difference between the two groups. The median age in the trial was 68 years, with $62.8 \%$ men, so again the population differed in the study population with older and more men than in our study. These discrepant findings between the CABANA trial and our study could, therefore, be explained by both differences in cohort selection and difference in definition of endpoints.

\section{Comorbidities and likelihood of catheter ablation in HF}

Based on data from our administrative registries, we identified clinical variables associated with the probability of undergoing catheter ablation. Being a younger male without comorbidities with a previous admission for HF seems to be the phenotype that is most likely to receive this treatment in Denmark. Except for having HF diagnosed in-hospital, all variables associated with being ablated were also associated with a better outcome. This should be kept in mind when interpreting our analyses since confounding by indication can never be eliminated in an observational study. In theory, the real-life effect of catheter ablation maybe even smaller.

\section{Methodological considerations}

The strength of the present study is the inclusion of $>10.000$ consecutive patients $\leq 75$ years of age, all treated with anticoagulation without anyone lost to follow-up during a long period. The rationale for the exclusion of patients $>75$ years was avoiding controls with a low likelihood of being offered AF catheter ablation. ${ }^{18}$ Investigating the two phenotypically different patient types $\mathrm{AF}$ before $\mathrm{HF}$ and $\mathrm{HF}$ before $\mathrm{AF}$ also contributes with new information regarding benefits of catheter ablation, since no significant difference in ATE was found between the two groups; hence our hypothesis regarding the larger benefit of catheter ablation in the AF before HF groups was not supported by the data. A high specificity of the used diagnostic codes in our registry and vital status on all patients are also strengths. ${ }^{12}$

\section{Limitations}

The present study is based on administrative codes and misclassification of $\mathrm{HF}$ and $\mathrm{AF}$ can, therefore, not be fully excluded. Further, important echocardiographic and clinical variables used for referral of patients with HF to AF ablation is neither available, and we were not able to differentiate between patients with $\mathrm{HF}$ and reduced versus preserved ejection fraction. Our results should be evaluated with that in mind. Unmeasured confounding due to lack of information on important clinical variables such as functional class, NT-proBNP and left ventricular ejection fraction-and residual confounding-for example, renal function is estimated based on a diagnostic code for CKD-cannot be excluded in our study. Information about the burden of AF after catheter ablation is also lacking, and if AF was paroxysmal, persistent, long-time persistent or permanent was unknown. We have no information on catheter ablation procedure details, reason for referral, nor on how they have changed over time at different centres. Finally, our study is observational, and our findings are associations and not necessarily due to causality. However, considering all the mentioned strengths of our study, the observed treatment effect size is biologically plausible. It may give a realistic real-life estimate of the effect of AF catheter ablation in HF.

\section{CONCLUSIONS}

Catheter ablation in patients with AF and HF was associated with reduced risk of a composite endpoint of $\mathrm{HF}$ readmission, stroke and all-cause mortality. This was true in both patients with $\mathrm{AF}$ before $\mathrm{HF}$ and $\mathrm{HF}$ before $\mathrm{AF}$ at 1 year after index date, respectively. Clinical outcome trials are needed to explore if these findings are causal.

\section{Author affiliations}

${ }^{1}$ Department of Cardiology, Gentofte, Copenhagen University Hospital, Hellerup, Hovedstaden, Denmark

${ }^{2}$ Department of Biostatistics, University of Copenhagen, Denmark, Copenhagen, Hovedstaden, Denmark

${ }^{3}$ Department of Cardiology, Rigshospitalet, Copenhagen University Hospital, Copenhagen, Denmark

${ }^{4}$ Department of Cardiology, Hillerød, Copenhagen University Hospital, Copenhagen, Hovedstaden, Denmark

${ }^{5}$ Department of Cardiology, Herlev, Copenhagen University Hospital, Herlev, Hovedstaden, Denmark

\section{Twitter Jannik Langtved Pallisgaard @jannik @CopCard @Pallisgaard_MD}

Contributors JLP and MS helped in planning, conduction, reporting and were guarantors of the study. TAG and AM helped in planning and conduction. Reviewing was done by MLH, A-MS, AJ, FG, GHG, CT-P, PKJ, SLK and LK.

Funding The authors have not declared a specific grant for this research from any funding agency in the public, commercial or not-for-profit sectors.

Competing interests $\mathrm{FG}$ is an advisor for Abbott, Carmat; Corvia (unpaid) speaker: Novartis, Astra, Orion Pharma; and investigator for Alnylam.

Patient consent for publication Not required.

Ethics approval In Denmark, retrospective register studies do not require approval from the ethics committees. The Danish Data Protection Agency approved this study (ref. no.: 2007-58-0015/GEH-2014-016 I-Suite no.: 02734), and data were made available in an anonymised format such that specific individuals could not be identified.

Provenance and peer review Not commissioned; externally peer reviewed.

Data availability statement Data may be obtained from a third party and are not publicly available.

Open access This is an open access article distributed in accordance with the Creative Commons Attribution Non Commercial (CC BY-NC 4.0) license, which permits others to distribute, remix, adapt, build upon this work non-commercially, and license their derivative works on different terms, provided the original work is properly cited, appropriate credit is given, any changes made indicated, and the use is non-commercial. See: http://creativecommons.org/licenses/by-nc/4.0/.

ORCID iD

Jannik Langtved Pallisgaard http://orcid.org/0000-0002-8072-3318 


\section{REFERENCES}

1 Kotecha D, Piccini JP. Atrial fibrillation in heart failure: what should we do? Eur Heart J 2015;36:ehv513-3257.

2 Dan G-A, Martinez-Rubio A, Agewall S, et al. Antiarrhythmic drugsclinical use and clinical decision making: a consensus document from the European heart rhythm association (EHRA) and European Society of cardiology (ESC) Working group on cardiovascular pharmacology, endorsed by the heart rhythm Society (HRS), AsiaPacific heart rhythm Society (APHRS) and International Society of cardiovascular pharmacotherapy (ISCP). Europace 2018;20:731-2.

3 Kirchhof P, Benussi S, Kotecha D, et al. 2016 ESC guidelines for the management of atrial fibrillation developed in collaboration with EACTS. Eur Heart J 2016;37:2893-962.

4 MacDonald MR, Connelly DT, Hawkins NM, et al. Radiofrequency ablation for persistent atrial fibrillation in patients with advanced heart failure and severe left ventricular systolic dysfunction: a randomised controlled trial. Heart 2011;97:740-7.

5 Ganesan AN, Nandal S, Lüker J, et al. Catheter ablation of atrial fibrillation in patients with concomitant left ventricular impairment: a systematic review of efficacy and effect on ejection fraction. Heart Lung Circ 2015;24:270-80.

6 Jones DG, Haldar SK, Hussain W, et al. A randomized trial to assess catheter ablation versus rate control in the management of persistent atrial fibrillation in heart failure. J Am Coll Cardiol 2013;61:1894-903.

7 Khan MN, Jaïs P, Cummings J, et al. Pulmonary-vein isolation for atrial fibrillation in patients with heart failure. $N$ Engl J Med 2008;359:1778-85.

8 Di Biase L, Mohanty P, Mohanty S, et al. Ablation versus amiodarone for treatment of persistent atrial fibrillation in patients with congestive heart failure and an implanted device: results from the AATAC multicenter randomized trial. Circulation 2016;133:1637-44.

9 Marrouche NF, Brachmann J, Andresen D, et al. Catheter ablation for atrial fibrillation with heart failure. N Engl J Med 2018;378:417-27.

10 Roy D, Talajic M, Nattel S, et al. Rhythm control versus rate control for atrial fibrillation and heart failure. $N$ Engl J Med 2008;358:2667-77.

11 Packer M, Kowey PR. Building castles in the sky. Circulation 2018;138:751-3.

12 Schmidt M, Schmidt SAJ, Sandegaard JL, et al. The Danish National Patient Registry: a review of content, data quality, and research potential. Clin Epidemiol 2015;7:449-90.

13 Schmidt M, Pedersen L, Sørensen HT. The Danish Civil Registration System as a tool in epidemiology. Eur J Epidemiol 2014;29:541-9.

14 Hernan JM, Robins MA. Causal inference. Chapman \& Hall/CRC, 2018.

15 Benichou J, Gail MH. Estimates of absolute cause-specific risk in cohort studies. Biometrics 1990;46:813-26.

16 R Foundation for Statistical Computing RCT. R: a language and environment for statistical computing. R core team 2019. Available: https://www.R-project.org/ [Accessed 19 Aug 2019].

17 Packer DL, Mark DB, Robb RA, et al. Effect of catheter ablation vs antiarrhythmic drug therapy on mortality, stroke, bleeding, and cardiac arrest among patients with atrial fibrillation: the CABANA randomized clinical trial. JAMA 2019;321:1261-74.

18 Pallisgaard JL, Gislason GH, Hansen J, et al. Temporal trends in atrial fibrillation recurrence rates after ablation between 2005 and 2014: a nationwide Danish cohort study. Eur Heart J 2018;39:442-9. 